\title{
Circadian pitfalls in experimental designs employing food restriction
}

\author{
RALPH MISTLBERGER \\ Simon Fraser University, Burnaby, British Columbia, Canada
}

\begin{abstract}
Most physiological and behavioral processes exhibit prominent circadian (24-h) rhythms that maintain a stable phase relation to daily light-dark cycles by means of entrainment. These rhythms can introduce interpretative confounds in experimental paradigms if lighting schedules and circadian phase of testing are not sufficiently controlled. The present review seeks to highlight another potential circadian confound implicit in experimental designs that utilize foodrestriction schedules: Such feeding schedules markedly alter the timing of most daily rhythms of behavior and physiology. Evidence is reviewed that this reflects entrainment of a distinct foodentrainable circadian oscillator analogous to the light-entrainable circadian oscillator that has been localized within the hypothalamic suprachiasmatic nuclei. Entrainment of this oscillator may occur under any experimental condition in which food is ingested at a relatively fixed time of day. Lack of awareness of this entrainment process and how it may vary with the parameters of the feeding schedule can have serious ramifications, ranging from increased variance in dependent measures to the generation of spurious results and interpretations.
\end{abstract}

Circadian rhythms dominate the temporal organization of mammalian behavior and physiology. These rhythms are generated by a system of endogenous circadian oscillators that are coordinated by one or more master pacemakers (Rosenwasser \& Adler, 1986; Rusak, 1982). Their influence is manifest not only in the familiar daily alternation between sleep and wakefulness (or rest and activity), but also in virtually every physiological regulatory system as well as in "higher order" processes such as learning and memory. An appreciation of this temporal structure is clearly obligatory for establishing optimal protocols for studying most psychobiological phenomena.

However, it is not enough to acknowledge that a particular variable of interest displays a 24-h rhythm; one must also know how this rhythm is influenced by periodic environmental stimuli. For most circadian rhythms, the daily light-dark (LD) cycle exerts a powerful entraining influence; that is, both the phase and the period of the rhythm are controlled (within limits) by the LD cycle, in a way analogous to the phase and period control of a self-sustaining physical oscillator by an appropriate periodic input (zeitgeber, or time-giver). The implications for experimental design are obvious and have been pointed out by others many times; the LD cycle must be controlled, the experimental subject must be maintained under this cycle until stably entrained, and the behavioral or physiological measurements of interest must be taken at a known phase of the animal's thythm or at a number of phases around the circadian cycle. To permit meaning-

Address correspondence to Ralph Mistlberger, Department of Psychology, Simon Fraser University, Burnaby, British Columbia V5A 1S6, Canada. ful comparisons across studies, these procedural details must be reported. In a recent follow-up survey of several journals of animal behavior and physiology, Hobbs and Goldberg (1987) found that an increasing number of research reports do provide this information, although the percentage for some journals is still well below 100 .

The purpose of the present review is to attempt to raise the profile of a potential circadian confound that appears to have gone virtually unrecognized among those conducting psychobiological research. Although LD cycles are a primary zeitgeber for circadian rhythms, they are not the only zeitgeber. In many organisms, the timing of food availability also greatly affects the phase and period of daily rhythms. In the following discussion, evidence is reviewed that establishes that daily schedules of food availability influence circadian rhythms by a process of entrainment analogous to entrainment by LD cycles. Effects of different types of feeding schedules are noted. The implications of entrainment by food restriction are then examined by several widely used experimental paradigms and techniques.

\section{Anticipation of Daily Mealtime in the Rat: Regulation by Circadian Clocks}

Richter (1922) was the first to demonstrate that timed food availability can influence daily rhythms of behavior in the rat. Although Richter utilized constant light in his initial studies, the effects are more easily described for rats recorded under a standard 12:12 h LD cycle. Such rats display predominantly nocturnal rhythms of wheelrunning and ingestive behavior (Figure 1A). If food is restricted to the middle of the light period, nocturnal activity is usually attenuated and a new bout of activity emerges in the day (Figure 1B). This bout of activity typi- 


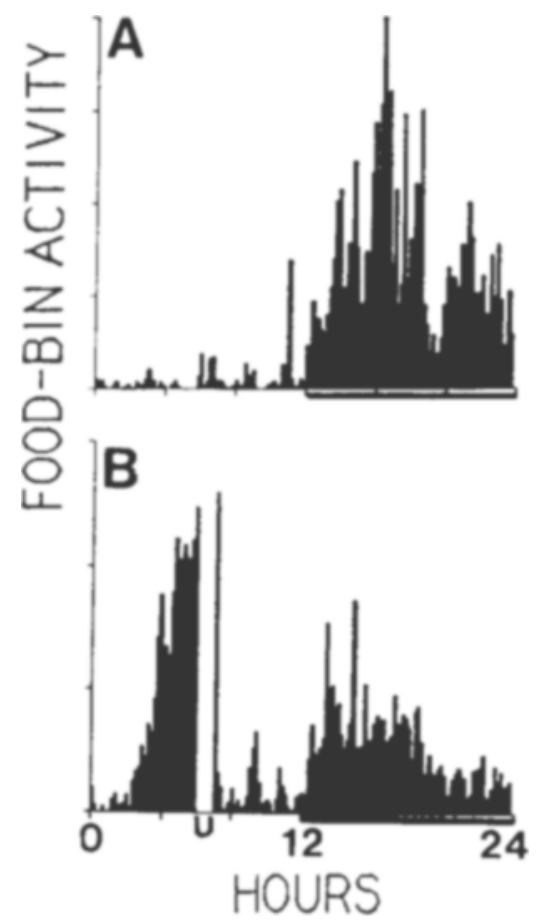

Figure 1. (A) Foodbin activity during each 10-min bin of the day averaged over 7 days of ad-lib food access for a single rat under LD 12:12. Lights-off is indicated by the heavy bar below the abscissa. (B) Foodbin activity of the same rat averaged over 7 days during which food was restricted to a single 1-h meal each day, indicated by the $U$ below the abscissa.

cally precedes the fixed daily mealtime by 1-3 h. It may appear within 2-4 days of food restriction and may take 1-2 weeks to stabilize (Figure 2A). In the simplest terms, the rat appears to "learn" the time of day that it will be fed.

More contemporary research has provided much evidence demonstrating that this form of learning has marked circadian constraints. Bolles and deLorge (1962) first observed that rats do not anticipate mealtime if food is provided every 19 or $29 \mathrm{~h}$. Stephan (1981) later established a range of $22-31 \mathrm{~h}$ within which a fixed daily meal schedule will induce food-anticipatory behavior. This circadian range of entrainment is comparable to, albeit somewhat broader than, that for LD cycles (Stephan, 1983). Stephan (1984) also observed that if the daily meal was shifted to another time of day, the food-anticipatory activity bout did not shift to the new time immediately, but instead shifted in a series of 2-3-h jumps or "transient" cycles. A similar process of shifting by a series of transient cycles also characterizes the response of LDentrained rhythms to a shift of the LD cycle. Aschoff, von Goetz, and Honma (1983) found that the duration of the food-anticipatory bout (the phase relation between the onset of premeal activity and the onset of mealtime) varied with the periodicity of the feeding cycle: short feeding cycles (food every $23 \mathrm{~h}$ ) were associated with short an- ticipatory bouts, whereas longer cycles $(25 \mathrm{~h})$ were associated with longer anticipatory bouts (the amount of food consumed per $24 \mathrm{~h}$ was held constant). The phase position of LD-entrained rhythms also depends on the period of the LD cycle (or, more accurately, on the difference between the respective periods of the LD cycle and the endogenous rhythm). Many researchers have noted that the food-anticipatory bout of activity disappears rapidly when food is provided ad lib. However, Coleman, Harper, Clarke, and Armstrong (1982) and Rosenwasser, Pelchat, and Adler (1984) demonstrated that if the rat is food-deprived for several days a few weeks later, a bout of activity reappears at the time of day previously associated with meal feeding. This seems analogous to damping or masking of LD-entrained rhythms in bright light but their persistence ("free run") in dim light or darkness.

These functional characteristics of food anticipation are readily interpreted within the framework of oscillator theory. A fixed daily meal serves as a zeitgeber that entrains a biological oscillator regulating the timing of wheelrunning behavior (i.e., foraging-related behaviors). This oscillator has circadian limits to entrainment, can shift only a few hours per day (as predicted by the limited entrainment range), varies its phase angle of entrainment as the difference between its intrinsic periodicity and the period of the feeding cycle varies, and is self-sustaining under constant conditions of food deprivation (but is masked or uncoupled from behavior when there are no temporal constraints on food availability). Anticipation of daily mealtime would thus appear to represent learning in only the most general sense of the word, since its underlying mechanism of clock entrainment is probably quite different from that envisaged in most neurobiological models of learning.

As noted above, the oscillator properties of foodassociated rhythms of behavior are similar to those of LDassociated rhythms. However, in the rat, the putative oscillators entrained by daily feeding schedules appear to be functionally and anatomically separate from the oscillators entrained by daily LD cycles. A rat fed for $2 \mathrm{~h}$ in the middle of the light period exhibits considerable foodanticipatory behavior and some persisting nocturnal activity. If the rat is blinded or placed in constant dark or dim light, the nocturnal component of activity begins to free-run-that is, it assumes a stable period in the circadian range and begins to drift systematically with respect to external time (Boulos, Rosenwasser, \& Terman, 1980; Gibbs, 1979). The food-anticipatory bout, in contrast, remains coupled to mealtime. The rat thus can express two rhythmic components of behavior with distinct circadian periodicities. These rhythmic components must be controlled by different oscillators. Destruction of the suprachiasmatic nuclei (SCN), which are thought to function as a light-entrainable circadian pacemaker, eliminates the free-running, light-entrainable component of behavior, but does not affect the food-entrained component (Boulos 



Figure 2. (A) Actogram of foodbin activity of a single 3-monthold male rat. Each line represents a day, with time in 10-min bins plotted left to right. Vertical deflections from the zero-activity line are proportional to the amount of activity in that time bin. Food was withheld during days indicated by brackets. Blanks represent meal time during days in which the rat was restricted to a single 1-h meal. The heavy bar on the abscissa indicates the lights-out period. (B) Foodbin activity of a 23-month-old male rat during adlib feeding, food deprivation, and food restriction. (C) Wheelrunning activity of a female rat. Blank sections represent the 2-h period when the rat was provided with a supplemental meal of palatable, nutritive mash. Standard chow pellets were available ad lib. (D) Wheel-running activity of a female rat with supplementary access to a palatable, non-nutritive mash. Standard chow was available ad lib. et al., 1980; Stephan, Swann, \& Sisk, 1979). Neural substrates mediating food-anticipatory rhythms remain to be identified, but most hypothalamic nuclei do not appear to be necessary for their expression (Mistlberger \& Rechtschaffen, 1984; Mistlberger \& Rusak, 1988).

On rare occasions, a rat is observed whose free-running rhythm entrains to a daily feeding schedule. This is thought to reflect coupling between the food-entrainable oscillators and the oscillators generating light-entrainable, free-running rhythms (Stephan, 1986). This coupling may be sufficiently strong in some rats to maintain synchrony within their circadian system under conditions that normally favor dissociation of oscillatory components. Alternatively, entrainment of the free-running circadian component may reflect a weak sensitivity of its underlying oscillator to feedback from the daily bout of intense activity preceding daily mealtime (Mistlberger, 1989; see below for further discussion).

Most of the literature on food entrainment is limited to studies of the rat; relatively little is known about the effects of feeding schedules on circadian organization in other species. In some species, including the rabbit (Jilge, Hornicke, \& Stahle, 1987), the kowari (a predatory marsupial; O'Reilly, Armstrong, \& Coleman, 1986), and the C57BL/6J mouse (Abe, Kidd, Tsuji, \& Mano, 1989), daily feeding schedules may entrain a dissociable, foodentrainable oscillator, whereas in other species, such as the common squirrel (Chandrashekaran, 1982), the pigeon (Abe \& Sugimoto, 1987), and the CS mouse (Abe et al., 1989), feeding schedules appear to entrain the animal's entire circadian system. This may reflect species differences in the coupling strength among different oscillators sensitive to LD and feeding zeitgebers, or it may indicate a sensitivity of light-entrainable oscillators to nonphotic zeitgebers in some species.

\section{The Nature of the Zeitgeber}

Food-anticipatory rhythms are most readily entrained by one or two fixed daily opportunities to feed. Meal duration can vary from $0.5 \mathrm{~h}$ to about $6 \mathrm{~h}$. Food anticipation is also observed if rats receive a limited amount of food each day at the same time, such as that required to maintain an animal at $80 \%-85 \%$ of its normal body weight. Most of the food will be consumed within $6 \mathrm{~h}$; thus limited-amount schedules are also effectively limitedduration schedules.

Food deprivation facilitates food anticipation but is not a necessary condition. Rats with ad-lib access to regular solid rodent chow show anticipatory wheel running in response to a daily 2 -h opportunity to consume a sweetened, oily food (presumably highly palatable) (Figure 2C; Mistlberger \& Rusak, 1987). However, the length of time required for such anticipatory rhythms to emerge is greater, ranging from 6-23 days, than the 3-10 days observed under typical restriction schedules. Food anticipation does not emerge until a daily meal accounts for 
a relatively large proportion of the animal's total daily caloric intake. In one study, most free-feeding rats did not anticipate a 4-g daily palatable supplementary meal but did entrain to a 10-g supplemental meal (Figure 2C; Mistlberger \& Rusak, 1987). The nature of the calorie source does not seem to matter; rats will anticipate a daily 0.5 -h meal of protein, fat, or carbohydrate provided that the meal accounts for at least $30 \%$ of the daily caloric intake (Mistlberger, Houpt, \& Moore-Ede, in press-b). Free-feeding rats do not show anticipatory rhythms to nonnutritive meals, such as a sweetened, oily cellulose mixture, despite consuming up to $11 \mathrm{~g}$ per daily 2-h access time (Figure 2D; Mistlberger \& Rusak, 1987).

Few other potential zeitgebers for anticipatory rhythms have been examined. Rats have been reported to anticipate a daily opportunity to drink water (e.g., see Dhume \& Gogate, 1982), but this may be secondary to the effect of water restriction on the timing of their food intake; that is, rats that show anticipation of water access appear to be those that eat large meals when water is available (Mistlberger \& Rechtschaffen, 1985). Rats with an experimentally induced salt appetite have been observed to anticipate a daily opportunity to ingest salt. However, the conditions under which this occurs seem to be highly constrained; anticipation appears in lever-pressing but not wheel-running behavior, to uncued but not to environmentally cued salt access, and under an LD cycle but marginally or not at all in constant light (Rosenwasser, Schulkin, \& Adler, 1985, 1988). Moreover, the effects of salt restriction on the timing of food and water intake in these rats are unknown.

Two other studies suggest that whatever the physical stimuli are that can entrain anticipatory rhythms, they do not do so by a common effect on the animal's general arousal state. Rats forced to run in a high-speed treadmill or swim in water for $2 \mathrm{~h}$ each day showed no evidence of anticipating this daily event, despite its highly arousing nature (Mistlberger, 1989). Also, rats stressed at the same time each day show no change in the waveform of their daily corticosterone rhythm that would suggest a circadian effect similar to that of food restriction (Ottenweller, Pitman, \& Natelson, 1987).

\section{The Range of Rhythms \\ Entrained by Feeding Time}

The majority of physiological variables examined in the rat exhibit daily rhythms that can be synchronized by restricted feeding schedules (for references, see Boulos \& Terman, 1980). Most prominent of these are the rhythms of gastroenteric enzymes and hormones that participate in the regulation of ingestive, digestive, and metabolic processes (Suda \& Saito, 1979). For many physiological variables, the timing of food intake is the primary circadian zeitgeber; synchronization to LD cycles may occur only by virtue of the fact that the daily rhythm of spontaneous food intake is strongly nocturnal under freefeeding conditions. Food availability thus appears to entrain a circadian oscillator that ensures both optimal daily timing of food-seeking behavior and optimal utilization of nutrients ingested at a predictable circadian phase.

\section{Potential Circadian Confounds}

Food-restriction schedules are ubiquitous in the psychobiological literature. The following discussion will examine in very general terms the potential impact of these schedules in several common experimental paradigms.

Learning and memory. Time of day is an important variable in performance on learning and memory tasks (for review, see Colquhoun, 1981; Rusak, 1981). The rhythms of motivation and sensory processes that help shape these time-of-day effects are entrained by LD cycles but may also be affected by food-restriction schedules. Such restriction schedules are widely used in studies of animal cognition. Typically, the animal is placed on a limited-amount food-restriction schedule until its body weight declines to $85 \%$ of normal. The animal is then trained on a task for a small food reward, receiving a food supplement at the end of the daily session to maintain its weight at a desired level. If the animal is tested at roughly the same time each day, this imposed feeding schedule should entrain food-anticipatory circadian thythms. If daily training sessions are tightly scheduled to a fixed time of day, and/or if the restriction schedule is especially lean, robust food-anticipatory rhythms should develop quite rapidly, probably within a few days. If a test session is then scheduled a few hours early or late on a given day, it may fall outside the circadian window of physiological and behavioral food anticipation. This would be akin to scheduling a test session 1-2 $\mathrm{h}$ before light onset in an animal that is usually trained $1-2 \mathrm{~h}$ after light onset. At a minimum, such variability of the test phase should promote increased variance in performance measures. If training sessions for an animal are more loosely scheduled over a wide circadian window, the animal may show relatively weak anticipatory rhythms that take many weeks to emerge and stabilize. This may introduce a source of variability in performance that is manifest over a span of weeks or months. It clearly is not enough to specify in general terms whether experimental manipulations occur in the light or the dark; rather, it may be necessary to place special emphasis on their timing with respect to feeding schedules imbedded in the experimental protocol.

Ingestive behavior and gastrointestinal physiology. Ingestive and gastrointestinal processes exhibit strong circadian modulation. Meal size and frequency vary with the phase of the LD cycle (Rosenwasser, Boulos, \& Terman, 1981). Following a single period of food deprivation, meal size depends on both the duration of deprivation and the circadian timing of meal initiation (Bellinger \& Mendel, 1975); that is, food intake reflects concurrent demands of both homeostatic and circadian regulatory processes. When a rat is limited to a $2-\mathrm{h}$ meal provided at the same time each day, the size of the meal increases for at least 7 days (Rowland, 1975), and in some cases for 14 days or more, before stabilizing (Mistlberger, unpublished observations). This parallels the rate of entrain- 
ment of gastroenteric enzyme rhythms by daily feeding schedules (Suda \& Saito, 1979). Other food-entrainable physiological rhythms that may influence feeding parameters include those of insulin release (Hara \& Saito, 1980) and duodenal motility (Comperatore \& Stephan, 1987). Meal size may increase much more slowly under more specialized feeding schedules, such as those involving daily supplements of a specific nutrient or palatable food in otherwise free-feeding animals. These prominent circadian characteristics of feeding behavior must be accounted for in the design and interpretation of any study of the neural basis and homeostatic properties of ingestive processes.

Neural activity. Circadian rhythms are widespread within the central nervous system (CNS). Relatively few studies have examined the influence of daily feeding schedules on these rhythms, but those that have report strong effects. Robust circadian rhythms of multiple-unit activity preceding daily feeding time have been observed in the lateral hypothalamus of food-entrained rats (Kurumiya \& Kawamura, 1985). Anticipatory changes have also been reported in the ventromedial hypothalamus, although these are weaker and inconsistent across studies (Inouye, 1983; Kurumiya \& Kawamura, 1985). Daily rhythms of $\alpha_{2}{ }^{-}$and $\beta$-adrenergic receptor binding within the hypothalamus correlate with feeding time in the rat (Krauchi, Wirz-Justice, Morimasa, Willener, \& Feer, 1984), as does the daily peak of endogenous noradrenaline release within the paraventricular hypothalamus (Leibowitz, 1988). Changes of noradrenaline and dopamine turnover have been noted in several hypothalamic regions as a function of food deprivation and refeeding (Jhanwar-Uniyal, Darwish, Levin, \& Leibowitz, 1987), and daily rhythms of serotonin and dopamine concentration in several brain regions are markedly altered in rats maintained on a 2-h diurnal feeding schedule (Krieger, Crowley, O'Donohue, \& Jacobowitz, 1980). A recent study of rats entrained to 2-h daily meals detected prominent food-anticipatory rhythms of dopamine release in the accumbens and caudate nuclei (Blaha, Mistlberger, \& Phillips, unpublished observations).

Such rhythms of neural activity may be problematic for some experimental designs. For example, learning of a specific task may parallel entrainment of an animal's circadian system by a feeding schedule implicit in the training protocol. A CNS variable (such as dopamine release) exhibiting changes over the course of training may appear to represent a correlate of task learning, but may instead be functionally related to the process of entrainment to feeding time. More generally, the existence of daily rhythms of neural activity that gradually entrain to feeding time requires that baseline values of neural activity prior to experimental manipulations be established only when food entrainment has clearly stabilized.

Pharmacology. Circadian rhythms of drug efficacy and toxicity are well documented (for review, see Moore-Ede, 1973; Reinberg, 1986). These presumably reflect daily rhythms of drug absorption, metabolism, and clearance. Changes in the timing of these rhythms as a consequence of food-restriction schedules can be expected. Thus, it is conceivable that the dose-response curve for a drug may vary depending on the time of administration with respect to a fixed daily mealtime. Also, greater variance in the dose-response relationship will occur if data are pooled from tests made before and after food entrainment has stabilized.

Specific neurochemical mediators of food-entrained oscillators are unknown. Presumably, drugs exist that affect these oscillators. Acute administration of such a drug might acutely shift the phase food-entrained rhythms. Daily administration of such a drug might activate and entrain these oscillators, thereby introducing potential circadian variability of drug effects in the absence of any feeding schedule. A striking example of this is the effect of chronic methamphetamine administered to $\mathrm{SCN}$-ablated rats; these rats are arrhythmic under ad-lib feeding schedules, but exhibit robust free-running circadian rhythms in response to methamphetamine ingested with water or released tonically from implanted minipumps (K. Honma, S. Honma, \& Hiroshige, 1987). This rhythm is food-entrainable but not light-entrainable; it may reflect activation of foodentrainable oscillators in the absence of a feeding zeitgeber (S. Honma, K. Honma, \& Hiroshige, 1989).

Ablation studies. Neural ablation may affect entrainment to feeding schedules in several ways. A particular lesion may destroy a food-entrainable pacemaker, it may alter the periodicity and thus the entrained phase of such a pacemaker, or it may sever afferent pathways mediating entrainment of the pacemaker or efferent pathways mediating coupling among multiple circadian oscillators and effector systems. Ablation may also change the type of anticipatory behavior that an animal exhibits prior to mealtime. For example, large medial hypothalamic lesions involving the paraventricular nuclei were associated with loss of food-anticipatory wheel-running rhythms but not with anticipatory foodbin approaches (Mistlberger \& Rusak, 1988).

Ablation studies designed to localize neural substrates of particular behaviors and regulatory functions in foodrestricted animals are not uncommon. For example, a study may examine the effects of a specific lesion on hyperactivity associated with food restriction. Differences between ablated and sham animals in such behaviors may potentially be secondary to altered entrainment to the feeding schedule employed.

Aging. Entrainment of food-anticipatory circadian rhythms changes with age. Richter (1922) reported attenuation or loss of food-anticipatory wheel-running in 2-yearold rats. In a more recent study, 22-24-month-old rats showed a delayed appearance and greatly reduced amplitude of food-anticipatory rhythms in response to a 2-h daily meal (Figure 2B; Mistlberger, Houpt, \& MooreEde, in press-a). Aging studies that compare behavior and physiology in young and old rats on restricted feeding 
schedules (e.g., as in learning and memory studies) could produce observed differences that are secondary to altered circadian function in aged animals.

\section{Other Nonphotic Zeitgebers: Physical Activity}

This review has focused on methodological issues associated with the use of restricted feeding schedules. However, it is worth noting that circadian rhythms in some species are sensitive to other nonphotic stimuli. One such stimulus is physical activity; free-running rhythms of hamsters can be phase-shifted by a single appropriately timed bout of wheel-running (Mrosovsky, 1988; Van Reeth \& Turek, 1989) and can be stably entrained by a daily bout of activity induced by cage changes (Mrosovsky, 1988; Reebs \& Mrosovsky, 1989) or restricted access to a foraging area (Rusak, Mistlberger, Losier, \& Jones, 1989). Unlike food entrainment in rats, physical activity in hamsters appears to entrain the entire free-running rhythm and not an independent "anticipatory" component. This may reflect strong coupling between light-entrainable oscillators and a distinct arousal-sensitive oscillator comparable to the rat's food-entrainable oscillator. Alternatively, it may indicate a direct sensitivity of the hamster's lightentrainable oscillator to nonphotic zeitgebers, with no intervening arousal-sensitive oscillator. In the absence of phenomenological or physiological evidence for such an oscillator, the latter alternative seems the more parsimonious interpretation at present. Food-restriction studies have not provided this evidence; hamsters do not hyperphage following food deprivation and thus do not survive, let alone entrain, under schedules of temporally restricted food availability (Silverman \& Zucker, 1976).

It is not yet known whether physical activity will greatly affect the phase of circadian rhythms of hamsters under conditions of entrainment to LD cycles. This needs to be explored before recommendations can be made concerning possible circadian confounds in experiments that require arousal of hamsters at a relatively fixed time each day. However, examination of the phase-response curve for physical activity suggests that there are circadian phases at which induced physical activity should have minimal circadian effects (see Mrosovsky, 1988).

Are other species sensitive to circadian effects of induced physical activity? In addition to hamsters, only mice and rats have been studied. Free-running rhythms of mice are apparently readily entrained by restricted access to a running wheel (D. M. Edgar, personal communication, 1988). By contrast, the circadian system of the rat seems largely insensitive to induced bouts of physical activity; as noted earlier, the free-running rhythm of the rat rarely entrains to a restricted feeding time, despite the intense 1-3-h bout of activity preceding the daily meal (Stephan, 1986). A daily 2 -h bout of forced treadmill running or swimming is similarly ineffective; only 1 animal of 18 tested exhibited entrainment to such schedules (Mistlberger, 1989). A mechanism for entrainment by activity thus does exist in rats, but its effects are so weak that in- duced activity seems unlikely to represent a functionally meaningful zeitgeber or a significant experimental confound in this species. Other species have yet to be studied, but it is reasonable to suspect that effects on activity and arousal states may explain some reported instances of entrainment by social cues (see Reebs, 1989).

\section{Conclusion}

Feeding schedules, like LD cycles, have a powerful effect on the timing of most biological rhythms. For many processes, feeding schedules are likely to be preeminent among potential environmental zeitgebers, including the LD cycle. Ignorance of this influence during the design and execution of experiments employing food restriction may introduce unnecessary variability in dependent measures and, in the worst cases, may promote spurious results and interpretations. To avoid these pitfalls, researchers using chronic food restriction should attempt to control the timing of feeding schedules and experimental testing with as much precision as possible. Knowledge of the time required for the emergence and stabilization of food-anticipatory rhythms would also seem important. As noted in the above discussion, this may vary widely with such factors as the type of feeding schedule employed and the age of the animal, as well as the species and dependent variables of interest. Precise scheduling of daily feeding and testing may not always be possible in experiments involving large numbers of animals and nonautomated, labor-intensive procedures. However, to assist the reader in the evaluation and replication of experimental results, it is incumbent on the researcher to report the temporal details of scheduling as accurately as possible, even when these details are perhaps less than optimal.

\section{REFERENCES}

Abe, H., Kida, M., Tsujl, K., \& Mano, T. (1989). Feeding cycles entrain circadian rhythms of locomotor activity in CS mice but not in C57BL/6J mice. Physiology \& Behavior, 45, 397-401.

AвE, H., \& Sugimoto, S. (1987). Food-anticipatory response to restricted food access based on the pigeon's biological clock. Animal Learning \& Behavior, 15, 353-359.

Aschoff, J., von Goetz, C., \& Honma, K. (1983). Restricted feeding in rats: Effects of varying feeding cycles. Zeitschrift fur Tierpsychologie, 63, 91-111.

BelLinger, L. L., \& MENDEL, V. E. (1975). Effect of deprivation and time of refeeding on food intake. Physiology \& Behavior, 14, 43-46.

BOLLES, R. C., \& DELORGE, J. (1962). The rat's adjustment to a-diumal feeding cycles. Journal of Comparative \& Physiological Psychology, $55,760-762$.

Boulos, Z., Rosenwasser, A. M., \& Terman, M. (1980). Feeding schedules and the circadian organization of behavior in the rat. $B e$ havioral Brain Research, 1, 39-65.

Boulos, Z., \& Terman, M. (1980). Food availability and daily biological rhythms. Neuroscience \& Biobehavioral Reviews, 4, 119-131.

Chandrashekaran, M. K. (1982). Social cues and circadian thythms. Current Science, 51, 158-167.

Coleman, G. J., Harper, S., Clarke, J. D., \& Armstrong, S. (1982). Evidence for a separate meal-associated oscillator in the rat. Physiology \& Behavior, 29, 107-115.

ColQunoun, P. (1981). Rhythms in performance. In J. Aschoff (Ed.), Handbook of behavioral neurobiology: 4. Biological rhythms (pp. 333350). New York: Plenum. 
Comperatore, C. A., \& Stephan, F. K. (1987). Entrainment of duodenal activity to periodic feeding. Journal of Biological Rhythms, 2, 227-242.

Dhume, R. A., \& Gogate, M. G. (1982). Water as entrainer of circadian running activity in rat. Physiology \& Behavior, 28, 431-436.

Gises, F. P. (1979). Fixed interval feeding does not entrain the circadian pacemaker in blind rats. American Journal of Physiology, 237, 249-253.

HARA, E., \& SAIto, M. (1980). Diurnal changes in plasma glucose and insulin responses to oral glucose load in rats. American Journal of Physiology, 238, 463-466.

HobBs, S. H., Goldberg, S. J. (1987). Circadian rhythms and animal behavior research: A follow-up survey. Psychobiology, 15, 192-194.

Honma, K., Honma, S., \& Hiroshige, T. (1987). Activity rhythms in the circadian domain appear in suprachiasmatic nuclei lesioned rats given methamphetamine. Physiology \& Behavior, 40, 767-774.

Honma, S., Honma, K., \& Hiroshige, T. (1989). Methamphetamine induced locomotor rhythm entrains to restricted daily feeding in SCN lesioned rats. Physiology \& Behavior, 45, 1057-1065.

INOUYE, S. T. (1983). Does the ventromedial hypothalamic nucleus contain a self-sustained circadian oscillator associated with periodic feedings? Brain Research, 279, 53-63.

Jhanwar-Uniyal, M., Darwish, M., Levin, B. E., \& Leibowitz, S. F. (1987). Alterations of catecholamine levels and turnover in discrete brain areas after food deprivation. Pharmacology, Biochemistry \& Behavior, 26, 271-275

Jilge, B., Hornicke, H., \& Stahle, H. (1987). Circadian rhythms of rabbits during restrictive feeding. American Journal of Physiology, 253, 46-54.

Krauchi, K., Wirz-Justice, A., Morimasa, T., Willener, R., \& FEER, H. (1984). Hypothalamic alpha-2 and beta-adrenoceptor rhythms are correlated with circadian feeding: Evidence from chronic methamphetamine treatment and withdrawal. Brain Research, 321, 83-90.

KRIEGER, D. T., CroWley, W. R., O'DonohUE, T. L., JACOBowITZ, D. M. (1980). Effects of food restriction on the periodicity of corticosteroids in plasma and on monoamine concentrations in discrete brain nuclei. Brain Research, 188, 167-174.

Kurumiya, S., \& Kawamura, H. (1985). Damped oscillation of the lateral hypothalamic multiple unit activity induced by restricted feeding schedules in rats with suprachiasmatic nucleus lesions. Society for Neuroscience Abstracts, 11, 817 .

LEIBowITZ, S. F. (1988). Hypothalamic paraventricular nucleus: Interaction between $\alpha 2$-noradrenergic system and circulating hormones and nutrients in relation to energy balance. Neuroscience \& Biobehavioral Reviews, 12, 101-109.

MistLBERGER, R. E. (1989). Effects of daily schedules of forced treadmill running or swimming on free-running rhythms in the rat. Manuscript submitted for publication.

Mistlberger, R. E., Houpt, T. A., \& Moore-Ede, M. C. (in press-a). The effects of aging on food-entrained circadian thythms in the rat. Neurobiology of Aging.

Mistlberger, R. E., Houpt, T. A., \& MoOre-Ede, M. C. (in press-b). Food-anticipatory rhythms under $24 \mathrm{~h}$ schedules of limited access to single macronutrients. Journal of Biological Rhythms.

Mistlberger, R., Rechtschaffen, A. (1984). Recovery of anticipatory activity to restricted feeding in rats with ventromedial hypothalamic lesions. Physiology \& Behavior, 33, 227-235.

Mistlberger, R., Rechtschaffen, A. (1985). Periodic water availability is not a potent zeitgeber for entrainment of circadian locomotor thythms in rats. Physiology \& Behavior, 34, 17-22.

Mistlberger, R., \& RuSaK, B. (1987). Palatable daily meals entrain anticipatory activity rhythms in free-feeding rats: Dependence on meal size and nutrient content. Physiology \& Behavior, 41, 219-226.

Mistlberger, R., \& RUSAK, B. (1988). Food anticipatory circadian rhythms in rats with paraventricular and lateral hypothalamic lesions. Journal of Biological Rhythms, 3, 277-291.

Moore-Ede M. C. (1973). Circadian rhythms of drug effectiveness and toxicity. Clinical Pharmacology \& Therapeutics, 14, 925-935.

Mrosovsky, N. (1988). Phase response curves for social entrainment. Journal of Comparative Physiology, A162, 35-46.

O'Reilly, H., Armstrong, S. M., \& Coleman, G. J. (1986). Restricted feeding and circadian activity rhythms of a predatory marsupial, Dasyuroides byrnei. Physiology \& Behavior, 38, 471-476.

Ottenweller, J. E., Pitman, D. L., Natelson, B. H. (1987). Repeated stress at the same time of day does not mimic timed feeding in its effects on the plasma corticosterone rhythm in rats. Chronobiologia, 14, 1-6.

REEBS, S. G. (1989). Acoustical entrainment of circadian rhythms in house sparrows: Constant light is not necessary. Ethology, 80, 172-181.

ReEBS, S. G., \& MrosovsKy, N. (1989). Effects of induced wheelrunning on the circadian activity rhythms of Syrian hamsters: Entrainment and phase response curve. Journal of Biological Rhythms, 4 , $39-48$.

ReINBerg, A. (1986). Circadian rhythms in effects of hypnotics and sleep inducers. International Journal of Clinical Pharmacology \& Research, 6, 33-44

RiCHTER, C. P. (1922). A behavioristic study of the activity of the rat. Comparative Psychology Monographs, 1, 1-55.

Rosenwasser, A. M., \& AdLer, N. T. (1986). Structure and function in the circadian timing systems: Evidence for multiple coupled circadian oscillators. Neuroscience \& Biobehavioral Reviews, 10, 431-448.

Rosenwasser, A. M., Boulos, Z., \& Terman, M. (1981). Circadian organization of food intake and meal patterns in the rat. Physiology \& Behavior, 27, 33-39.

Rosenwasser, A. M., Pelchat, R. J., \& Adler, N. T. (1984). Memory for feeding time: Possible dependence on coupled circadian oscillators. Physiology \& Behavior, 32, 25-30.

Rosenwasser, A. M., Schulkin, J., \& Adler, N. T. (1985). Circadian wheel-running activity of rats under schedules of limited daily access to salt. Chronobiology International, 2, 115-119.

Rosenwasser, A. M., Schulkin, J., \& Adler, N. T. (1988). Anticipatory appetitive behavior of adrenalectomized rats under circadian saltaccess schedules. Animal Learning \& Behavior, 16, 324-329.

RoWLAND, N. (1975). Feeding patterns in rats on restricted access schedules: Palatability, bulk, and other determinants of intake. Bulletin of the Psychonomic Society, 5, 306-308.

RusAK, B. (1981). Vertebrate behavioral rhythms. In J. Aschoff (Ed.), Handbook of behavioral neurobiology: 4. Biological thythms (pp. 183213). New York: Plenum.

RusAK, B. (1982). Physiological models of the rodent circadian system. In J. Aschoff, S. Daan, \& G. A. Groos (Eds.), Vertebrate circadian systems (pp. 62-73). New York: Springer-Verlag.

Rusak, B., Mist lberger, R. E., Losier, B., \& Jones, C. H. (1989). Daily hoarding opportunity entrains the pacemaker for hamster activity rhythms. Journal of Comparative Physiology, A164, 165-171.

Silverman, H. J., \& ZuCkER, I. (1976). Absence of post-fast food compensation in the golden hamster (Mesocricetus auratus). Physiology \& Behavior, 17, 271-285.

STEPHAN, F. K. (1981). Limits of entrainment to periodic feeding in rats with suprachiasmatic lesions. Journal of Comparative Physiology, 143, 401-410.

Stephan, F. K. (1983). Circadian rhythms in the rat: Constant darkness, entrainment to T cycles and to skeleton photoperiods. Physiol. ogy \& Behavior, 30, 451-462.

Stephan, F. K. (1984). Phase shifts of circadian rhythms in activity entrained to food access. Physiology \& Behavior, 32, 663-671.

StePhan, F. K. (1986). Coupling between feeding and light-entrainable circadian pacemakers in the rat. Physiology \& Behavior, 38, 537-544.

Stephan, F. K., SwanN, J. M., \& SiSK, C. L. (1979). Entrainment of circadian rhythms by feeding schedules in rats with suprachiasmatic nucleus lesions. Behavioral \& Neural Biology, 25, 545-554.

SUdA, M., \& SAITo, M. (1979). Coordinative regulation of feeding behavior and metabolism by a circadian timing system. In $M$. Suda, O. Hayaishi, \& H. Nakagawa (Eds.), Biological rhythms and their central mechanism (pp. 263-271). New York: Elsevier North-Holland.

VAN REETH, O., \& TUREX, F. W. (1989). Stimulated activity mediates phase shifts in the hamster circadian clock induced by dark pulses or benzodiazepines. Nature, 339, 49-51.

(Manuscript received October 26, 1989; revision accepted for publication January 10, 1990.) 